\title{
Calcium, vitamin D, casein and whey protein intakes and periodontitis among Danish adults
}

\author{
Amanda RA Adegboye ${ }^{1,2, *}$, Barbara J Boucher ${ }^{3}$, Johanne Kongstad ${ }^{4}$, Nils-Erik Fiehn ${ }^{5}$, \\ Lisa B Christensen ${ }^{4}$ and Berit L Heitmann ${ }^{1,6,7}$ \\ 'Research Unit for Dietary Studies, Institute of Preventive Medicine, Frederiksberg and Bispebjerg Hospital, \\ Copenhagen University, Copenhagen, Denmark: ${ }^{2}$ Department of Life Sciences, University of Westminster, $115 \mathrm{New}$ \\ Cavendish Street, C1.43, London WIW 6UW, UK: ${ }^{3}$ Centre for Diabetes, Bart's \& The London School of Medicine \& \\ Dentistry, Queen Mary University of London, Blizard Institute, London, UK: ${ }^{4}$ Section for Periodontology and \\ Community Dentistry, Department of Odontology, Faculty of Health and Medical Science, University of Copenhagen, \\ Copenhagen, Denmark: ${ }^{5}$ Department of International Health, Immunology and Microbiology, Faculty of Health and \\ Medical Sciences, University of Copenhagen, Copenhagen, Denmark: ${ }^{6} T$ he Boden Institute of Obesity, Nutrition, \\ Exercise \& Eating Disorders, The University of Sydney, Sydney, New South Wales, Australia: ${ }^{7}$ The National Institute \\ of Public Health, University of Southern Denmark, Copenhagen, Denmark
}

Submitted 3 October 2014: Final revision received 1 February 2015: Accepted 11 March 2015: First published online 4 May 2015

\begin{abstract}
Objective: To investigate whether intakes of $\mathrm{Ca}$, vitamin $\mathrm{D}$, casein and whey are associated with periodontitis and to investigate the possibility of interactions between them.

Design: Cross-sectional study. An Internet-based, 267-item FFQ was used to assess dietary intake. Intakes of casein $(32.0 \mathrm{~g} / \mathrm{d})$, whey proteins $(9.6 \mathrm{~g} / \mathrm{d})$ and vitamin D $(5 \cdot 8 \mu \mathrm{g} / \mathrm{d})$ were classified as within $v$. above the 50 th percentile. Ca intake was classified as within $v$. below age-specific recommendations. Severe periodontitis was defined as having $\geq 2$ inter-proximal sites with clinical attachment loss $\geq 6 \mathrm{~mm}$ (not on the same tooth) and $\geq 1$ inter-proximal site with pocket depth $\geq 5 \mathrm{~mm}$. Since vitamin $\mathrm{D}$ influences $\mathrm{Ca}$ absorption, models were stratified by lower and higher $(<5.8 v . \geq 5 \cdot 8 \mu \mathrm{g} / \mathrm{d})$ vitamin $\mathrm{D}$ intake.

Setting: Danish Health Examination Survey (DANHES) 2007-2008.

Subjects: Adult participants ( $n$ 3287) in the oral health study of DANHES 2007-2008.

Results: Intakes of $\mathrm{Ca}$ within recommendations ( $\mathrm{OR}=0.76 ; 95 \% \mathrm{CI} 0.58,0.99)$, whey $\geq 9.6 \mathrm{~g} / \mathrm{d}(\mathrm{OR}=0.75 ; 95 \%$ CI $0.58,0.97)$ and casein $\geq 32 \mathrm{~g} / \mathrm{d}(\mathrm{OR}=0.75$ $95 \%$ CI $0.58,0.97)$ were associated with lower likelihood of severe periodontitis after adjustment for age, gender, education, smoking, sucrose intake, alcohol consumption, number of teeth, daily brushing, regular visits to the dentist and chronic illness, irrespective of vitamin D intake levels. Intake of vitamin D alone was not associated severe with periodontitis.

Conclusions: Intakes of $\mathrm{Ca}$, casein and whey protein were inversely associated with periodontitis. Consumption of foods rich in $\mathrm{Ca}$, casein and whey (e.g. dairy foods) should be promoted, as they may contribute to the prevention of periodontitis. Further longitudinal studies are required to confirm these associations.
\end{abstract}

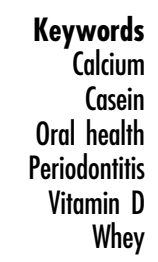

Periodontitis is a bacterially induced chronic inflammation affecting the supporting structures of teeth; it is characterized by periodontal attachment loss and bone loss, which can subsequently lead to tooth loss ${ }^{(1)}$. The pathogenesis of periodontitis involves an ecological shift in the biofilm leading to bacterial activation of immune-inflammatory mechanisms, of which several have adverse effects on bone and connective tissue remodelling ${ }^{(2)}$.
Research points towards potential risk factors for periodontal diseases that lie well beyond the mouth, including hormonal changes, diabetes, stress, genetic susceptibility, smoking, alcohol consumption and other lifestyle factors ${ }^{(3-5)}$. Eating habits and a low intake of specific nutrients have also been found to be related to periodontal diseases ${ }^{(6,7)}$.

Dietary factors may impact periodontitis risk and overall oral health via systemic or local effects. Diet can have 
systemic effects when absorbed nutrients and minerals affect tooth development and growth, or in relation to the individual's resistance to periodontitis and caries ${ }^{(8)}$. In addition, diet may affect the virulence of certain microorganisms $^{(8)}$. Local effects include the influence of diet on dental plaque, which is the primary aetiological factor for the initiation of periodontal disease, and the amount, composition and acidity of the saliva ${ }^{(9,10)}$, factors that also affect periodontitis risk.

Although healthy eating habits seem to be protective against periodontal diseases ${ }^{(11)}$, original research on nutritional determinants of periodontitis is still scarce. To date, few studies have investigated the importance of interactions between dietary nutrients in the development and progression of periodontal diseases and tooth loss ${ }^{(12)}$. Most studies have investigated the effect of single nutrients on periodontal disease. However, minerals, vitamins and other nutrients have interactions between them that affect their functionality, availability or absorption ${ }^{(13)}$.

We have suggested previously that ingestion of dairy products (which are a good source of $\mathrm{Ca}$, vitamin $\mathrm{D}$, casein and whey protein) confers protection against periodontitis $^{(14)}$. High concentrations of $\mathrm{Ca}$ and phosphate in saliva may inhibit bacterial biofilm formation ${ }^{(15)}$; and high $\mathrm{Ca}$ intakes may be associated with prevention of alveolar bone loss and consequently better retention of natural dentition ${ }^{(16)}$. Vitamin D is essential for Ca absorption $^{(13)}$ and promotes oral health through its effects on bone metabolism and innate immunity ${ }^{(1)}$. Whey protein enhances immune system function and inhibits plaque formation $^{(17)}$. Indeed, we found earlier that higher dairy $\mathrm{Ca}$, but not non-dairy $\mathrm{Ca}$, was protective against tooth loss and periodontitis and was associated with lower plaque scores; furthermore, our findings suggested that sufficient vitamin $D$ in the diet was of importance for the appearance of the significant benefits of higher $\mathrm{Ca}$ intakes ${ }^{(18)}$. However, our previous results were based on analyses of data on small population subsets, limiting further exploration of potential interactions between periodontitis and dairy nutrients such as Ca, vitamin D or the dairy protein casein, or those in whey ${ }^{(14,18)}$. Therefore the present study aimed to investigate whether intakes of $\mathrm{Ca}$, vitamin $\mathrm{D}$, casein and whey are associated with periodontitis and to investigate the possibility of interactions between them in relation to periodontitis.

\section{Methods}

\section{Study design and population}

The Danish Health Examination Survey (DANHES 2007-2008) is a cross-sectional study carried out in thirteen of ninetyeight municipalities in Denmark. The focus of the survey was on diet, smoking, alcohol and physical activity ${ }^{(19)}$.

In total 538497 adults, aged 18 years or older, were invited to complete a basic Internet-based questionnaire concerning social factors, lifestyle and general health. A random sub-sample of these individuals ( $n 180$ 103) was also invited to participate in a general health examination and fill in a supplementary Internet-based FFQ. Out of 538497 individuals, 76484 completed the basic questionnaire and 18065 participated in the general health examination and fully or partially completed the supplementary FFQ (Fig. 1) ${ }^{(19)}$.

As a part of DANHES 2007-2008, an oral health study based on clinical examination and a separate questionnaire was carried out in 2008-2009. Participants were consecutively enrolled on a first come, first served basis. Among 18065 participants who completed the general health examination in 2007-2008, 4402 individuals volunteered to participate in the present oral health study $^{(20)}$ (Fig. 1). Participants from this subset ( $n$ 4402) and those participating in the general study ( $n$ 18065) did not differ with respect to age and gender. However, participants in the oral health study reported higher income and more years of education than non-participants in the oral health study group.

The study was conducted in accordance with the Helsinki Declaration and approved by the Ethical Committees for the Region of Copenhagen. Written informed consent was obtained from all participants.

\section{Dietary assessment}

An Internet-based, semi-quantitative, 267-item FFQ was used to assess dietary intake based on intake over the previous year ${ }^{(19)}$. The dietary questionnaire had previously been validated and used in many Danish population studies $^{(21)}$. In the present survey, the FFQ was extended with thirty-nine portion size items placed at the end of the questionnaire ${ }^{(22)}$. Each item referred to four or six size classes presented in a picture series developed by the Danish Veterinary and Food Administration.

Calculation of total energy $(\mathrm{kJ} / \mathrm{d})$ and dietary $\mathrm{Ca}(\mathrm{mg} / \mathrm{d})$ intakes, and the distribution of intake of fat, carbohydrate and protein, were performed using the program FoodCalc $^{\circledR}$ and Danish official food composition tables ${ }^{(23)}$. Due to limited information on Ca contents of the supplements used, $800 \mathrm{mg}$ was added to the total Ca intake, but only for those who reported daily intake of $\mathrm{Ca}$ supplements over the previous year. Total dietary and supplemental Ca intakes were classified as: below $v$. within recommendations $^{(24)}$. An intake of $1000 \mathrm{mg} \mathrm{Ca} / \mathrm{d}$ is recommended for female adults aged $<50$ years and males aged $<70$ years; an intake of $1200 \mathrm{mg} \mathrm{Ca} / \mathrm{d}$ is recommended for female adults aged $>50$ years and males aged $>70$ years.

Total dietary and supplemental intakes $(\mu \mathrm{g} / \mathrm{d})$ of ergocalcifierol (vitamin $\mathrm{D}_{2}$ ) and cholecalcifierol (vitamin $\mathrm{D}_{3}$ ) were estimated. For daily supplemental vitamin D or for the use of multivitamins, intakes of $10 \mu \mathrm{g}$ or $5 \mu \mathrm{g}$ vitamin D were added, respectively. Only $7 \cdot 8 \%$ of participants reported vitamin $\mathrm{D}$ intake within current recommendations $(15 \mu \mathrm{g} / \mathrm{d}$ for adults aged $51-70$ years and $20 \mu \mathrm{g} / \mathrm{d}$ for those 
aged $\geq 71$ years) ${ }^{(24)}$. Therefore, vitamin $\mathrm{D}$ intake was classified as within $v$. above the 50th percentile $(5 \cdot 8 \mu \mathrm{g} / \mathrm{d})$.

Dairy protein intake was calculated from all dairy foods, including dairy ingredients used in preparing various foods (for example, cheese in pizza and milk in mashed potatoes). For each dairy food the content of whey and casein proteins was estimated. Whey proteins make up about $20 \%$ of the protein content of milk, yoghurt, ice cream, butter and cream, the rest of the protein content being in the form of casein fractions $(\sim 80 \%)$. For most cheeses, the protein content is $100 \%$ casein, as whey proteins are eliminated after the curdling process from hard cheeses, but some are left in cottage cheese ${ }^{(25)}$. Intakes of casein $(32.0 \mathrm{~g} / \mathrm{d})$ and whey proteins $(9.6 \mathrm{~g} / \mathrm{d})$ were classified as within $v$. above the 50th percentile.

\section{Oral examination}

The participants filled in a questionnaire that asked validated questions about self-reported oral health status, oral health problems, utilization of dental care and oral hygiene habits.

The oral health study focused upon dental status, dental caries and erosion, the quantity and type of restorative treatments, periodontal disease, salivary flow rates, selfassessment of oral disease, oral health care habits and oral-health-related quality of life.

Standard dental equipment was used for the clinical oral examination. The manual periodontal probe included pagination at 2, 4, 6, 8, 10 and $12 \mathrm{~mm}$ (model 8-520B; LM-Instruments, Finland). The examination protocol was designed to minimize measurement errors. Three dental hygienists were responsible for the oral examination and they had been carefully trained by two experienced clinical examiners. At the beginning and at the end of the data collection the dental hygienists were revalidated. Inter- and intra-examiner agreement ranged between 60 and $80 \%$ for periodontal measurements ${ }^{(20)}$.

The periodontal examination included half-mouth (one upper and one lower quadrant randomly selected) records, except for third molars. The assessments comprising pocket depths (PD), bleeding on probing (BOP) and gingival margin levels were registered at six sites of each tooth measured. Gingival recession was recorded as a negative value. Clinical attachment loss (CAL) was calculated as the sum of the PD and gingival level measurements, for each site. The mean CAL, including and excluding mid-facial and mid-lingual sites, respectively, was calculated for each participant.

Severe periodontitis was defined according to the Centers for Disease Control and Prevention (CDC)/American Academy of Periodontology (AAP) criteria $^{(26)}$ as having $\geq 2$ inter-proximal sites with CAL $\geq 6 \mathrm{~mm}$ not on the same tooth and $\geq 1$ inter-proximal site with $\mathrm{PD} \geq 5 \mathrm{~mm}$ (yes/no).

\section{Covariates}

These included age, gender, years of education $(<10 v$. $\geq 10$ years), presence of any long-term and chronic illness (yes/no), regular visits to a dentist (at least once per annum for a check-up during the last 5 years, yes/no), daily tooth brushing (yes/no), number of remaining teeth, current smoking (yes/no) and alcohol intake (above $v$. within recommendations from the Danish National Board of Health: maximum consumption of 14 units alcohol/ week for women and 21 units alcohol/week for men) ${ }^{(27)}$. Sucrose consumption above $10 \%$ of total energy intake is considered the threshold that potentially increases caries rates and that is associated with lower consumption of vitamins and minerals ${ }^{(28)}$. In the study population, 148 individuals ( $4.5 \%$ ) presented sucrose consumption above $10 \%$ of total energy intake and of those, only one individual had severe periodontitis. Therefore, sucrose consumption adjusted for total energy intake $(\mathrm{g} / \mathrm{kcal})$ was entered in the models as a continuous variable.

\section{Analysis}

Characteristics of the study population are presented as mean and standard deviation or as percentage and number. Logistic regression and linear regression, adjusted for potential confounders, were used to evaluate associations of intakes of $\mathrm{Ca}$, vitamin $\mathrm{D}$, and casein and whey proteins with severe periodontitis and mean CAL, respectively. Effect modification by gender was evaluated. As no significant interaction was found, data analyses were not stratified by gender. The level of significance was set at $P$ value $<0.05$. All statistical analyses were performed using the statistical software package STATA version 9.2.

\section{Results}

Among 4402 participants, 1115 individuals were excluded due to incomplete dietary information and edentulism, leaving a final study population of 3287 individuals with complete information on diet and oral health (Fig. 1). Participants with complete information tended to be younger, more educated, less likely to smoke daily and have lower mean systolic blood pressure as compared with those excluded because of incomplete information $(P<0.05)$; but included and excluded subjects did not differ with respect to gender, presence of chronic illness, diabetes or regular visits to the dentist (data not shown).

The final study population was composed of $38.7 \%$ men and $61.3 \%$ women and their age ranged from 18 to 95 years (mean 52.7 (sD 13.4) years). Of the final study population, $9.6 \%$ had severe periodontitis. Descriptive data according to periodontitis status are presented in Table 1. Individuals presenting severe periodontitis were older, less educated, more likely to be male, to smoke daily and to have chronic illness, fewer teeth and higher mean CAL, compared with those without chronic and severe periodontitis, according to the CDC/AAP definition ${ }^{(26)}$. In addition, individuals with periodontitis were more likely to 


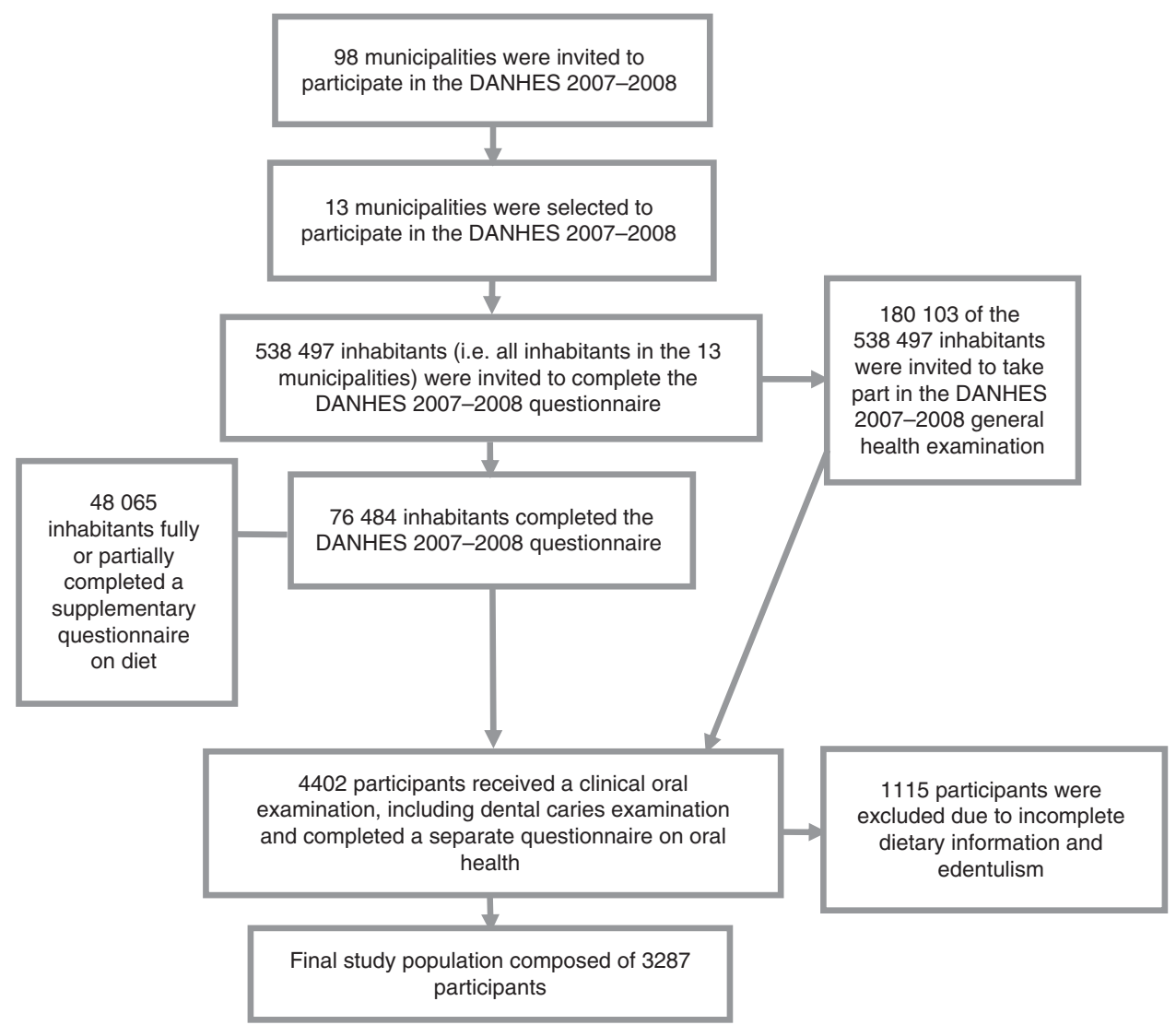

Fig. 1 Flowchart of participants in the Danish Health Examination Survey (DANHES 2007-2008)

Table 1 General characteristics of the study population according to severe periodontitis status; adult participants of the oral health study of the Danish Health Examination Survey 2007-2008

\begin{tabular}{|c|c|c|c|c|c|c|c|}
\hline \multirow[b]{3}{*}{ Characteristic } & & & \multicolumn{4}{|c|}{ Periodontitis status } & \multirow[b]{3}{*}{$P$} \\
\hline & \multicolumn{2}{|c|}{ Total study group } & \multicolumn{2}{|c|}{ No } & \multicolumn{2}{|c|}{ Yes } & \\
\hline & Mean or \% & SD or $n$ & Mean or $\%$ & SD or $n$ & Mean or \% & SD or $n$ & \\
\hline Age (years)* & $52 \cdot 7$ & $13 \cdot 4$ & $51 \cdot 8$ & $13 \cdot 4$ & 62.5 & $9 \cdot 1$ & $<0.0001$ \\
\hline$<10$ years of education $\dagger$ & $8 \cdot 8$ & 288 & 8.0 & 240 & $16 \cdot 2$ & 48 & $<0.0001$ \\
\hline Malest & 38.7 & 1273 & 37.5 & 1121 & $51 \cdot 2$ & 152 & $<0.0001$ \\
\hline Daily smoking $†$ & 8.7 & 287 & $7 \cdot 6$ & 226 & 20.5 & 61 & $<0.0001$ \\
\hline Vitamin D intake $<5.8 \mu \mathrm{g} / \mathrm{d} \dagger$ & $50 \cdot 0$ & 1645 & $50 \cdot 4$ & 1508 & $46 \cdot 1$ & 137 & 0.2 \\
\hline Vitamin D intake $(\mu \mathrm{g} / \mathrm{d})^{\star}$ & $7 \cdot 25$ & $5 \cdot 1$ & 7.5 & $5 \cdot 0$ & $7 \cdot 2$ & $5 \cdot 1$ & 0.3 \\
\hline $\mathrm{Ca}$ intake below recommendations $\dagger$ & $66 \cdot 7$ & 2194 & $66 \cdot 9$ & 2003 & $64 \cdot 3$ & 191 & 0.2 \\
\hline Ca intake $(\mathrm{mg} / \mathrm{d})^{\star}$ & 1084.9 & $567 \cdot 2$ & $1084 \cdot 6$ & $553 \cdot 8$ & $1087 \cdot 7$ & 689.3 & 0.9 \\
\hline Whey protein intake $<9.6 \mathrm{~g} / \mathrm{d} \dagger$ & $50 \cdot 3$ & 1655 & $49 \cdot 8$ & 1490 & 53.6 & 449 & 0.031 \\
\hline Whey protein intake $(\mathrm{g} / \mathrm{d})^{\star}$ & $10 \cdot 0$ & $4 \cdot 2$ & $10 \cdot 1$ & $4 \cdot 1$ & 9.8 & $5 \cdot 0$ & 0.2 \\
\hline Casein intake $<32.0 \mathrm{~g} / \mathrm{d} \dagger$ & $50 \cdot 1$ & 1649 & $50 \cdot 6$ & 1512 & $46 \cdot 1$ & 137 & 0.1 \\
\hline Casein intake $(g / d)^{\star}$ & $34 \cdot 2$ & $15 \cdot 0$ & $34 \cdot 2$ & $14 \cdot 7$ & $34 \cdot 8$ & $18 \cdot 1$ & 0.4 \\
\hline Sucrose intake $(\mathrm{g} / \mathrm{kcal}) \dagger$ & 4.7 & $2 \cdot 0$ & 4.8 & $2 \cdot 8$ & 3.9 & 1.7 & $<0.0001$ \\
\hline Alcohol above recommendations $\dagger$ & $11 \cdot 6$ & 383 & $11 \cdot 0$ & 330 & $17 \cdot 8$ & 53 & $<0.0001$ \\
\hline Chronic illnesst & $36 \cdot 5$ & 1200 & $36 \cdot 0$ & 1076 & 41.7 & 124 & 0.049 \\
\hline Regular visits to the dentist $†$ & $85 \cdot 8$ & 2821 & $85 \cdot 9$ & 2569 & 84.9 & 252 & 0.6 \\
\hline Daily tooth brushing $\dagger$ & $81 \cdot 7$ & 2686 & $81 \cdot 8$ & 2447 & $80 \cdot 5$ & 239 & 0.6 \\
\hline Number of teeth* ${ }^{*}$ & $27 \cdot 0$ & 3.8 & $27 \cdot 3$ & 3.6 & 24.7 & 4.8 & $<0.0001$ \\
\hline Mean CAL (including inter-proximal sites)* & $2 \cdot 3$ & 0.8 & $2 \cdot 1$ & 0.6 & 3.6 & $1 \cdot 2$ & $<0.0001$ \\
\hline Mean CAL (excluding inter-proximal sites) & $2 \cdot 2$ & 0.8 & $2 \cdot 1$ & 0.5 & 3.7 & 1.2 & $<0.0001$ \\
\hline
\end{tabular}

CAL, clinical attachment loss.

Analysis performed with 3287 observations ( $n 297$ for severe periodontitis).

*Data are presented as mean and standard deviation.

†Data are presented as percentage and number. 
Table 2 Associations of intakes of calcium, vitamin D, casein and whey with severe periodontitis among adult participants of the oral health study of the Danish Health Examination Survey 2007-2008

\begin{tabular}{|c|c|c|c|c|c|c|}
\hline & \multicolumn{3}{|c|}{ Crude } & \multicolumn{3}{|c|}{ Adjusted $^{*}$} \\
\hline & OR† & $95 \% \mathrm{Cl}$ & $P$ & OR† & $95 \% \mathrm{Cl}$ & $P$ \\
\hline $\mathrm{Ca}(\mathrm{mg} / \mathrm{d})$ & 1.00 & $0.99,1.00$ & 0.9 & 0.99 & $0.99,1.00$ & 0.4 \\
\hline Vitamin D $(\mu \mathrm{g} / \mathrm{d})$ & 1.00 & $0.99,1.03$ & 0.3 & 0.98 & $0.95,1.00$ & 0.1 \\
\hline Whey $(g / d) "$ & 0.98 & $0.95,1.00$ & 0.2 & 0.98 & $0.95,1.01$ & 0.2 \\
\hline Casein $(\mathrm{g} / \mathrm{d})$ & 1.00 & $0.99,1.01$ & 0.4 & 0.99 & $0.99,1.01$ & 0.8 \\
\hline Ca intake within recommendations & $1 \cdot 16$ & $0.90,1.48$ & 0.2 & 0.76 & $0.58,0.99$ & 0.041 \\
\hline Vitamin $D \geq 5.8 \mu \mathrm{g} / \mathrm{d}$ & 1.20 & $0.93,1.50$ & 0.2 & 0.85 & $0.66,1.01$ & 0.2 \\
\hline Whey $\geq 9.6 \mathrm{~g} / \mathrm{d}$ & 0.77 & $0.60,0.97$ & 0.031 & 0.75 & $0.58,0.97$ & 0.030 \\
\hline Casein $\geq 32.0 \mathrm{~g} / \mathrm{d}$ & 0.83 & $0.65,1.05$ & 0.1 & 0.75 & $0.58,0.97$ & 0.031 \\
\hline
\end{tabular}

*Logistic regression models adjusted for age, gender, education, smoking, sucrose intake, alcohol consumption, number of teeth, daily brushing, regular visits to the dentist and chronic illness.

†Odds ratio is for one-unit increase in Ca, vitamin D, casein or whey protein intake. Analysis performed with 3287 observations.

Table 3 Associations of intakes of intakes of calcium, vitamin D, casein and whey with mean clinical attachment loss (CAL) among adult participants of the oral health study of the Danish Health Examination Survey 2007-2008

\begin{tabular}{|c|c|c|c|c|c|c|}
\hline & \multicolumn{3}{|c|}{ CAL (including mid-facial \& mid-lingual sites) } & \multicolumn{3}{|c|}{ CAL (excluding mid-facial \& mid-lingual sites) } \\
\hline & Coefficient $^{*}$ & $95 \% \mathrm{Cl}$ & $P$ & Coefficient $^{*}$ & $95 \% \mathrm{Cl}$ & $P$ \\
\hline $\mathrm{Ca}(\mathrm{mg} / \mathrm{d})$ & -0.00002 & $-0.0006,0.00001$ & 0.3 & -0.00003 & $-0.0007,0.00001$ & 0.2 \\
\hline Vitamin D $(\mu \mathrm{g} / \mathrm{d})$ & 0.0008 & $-0.003,0.0053$ & 0.7 & 0.00005 & $-0.004,0.005$ & 0.9 \\
\hline Whey $(g / d)$ & -0.002 & $-0.007,0.003$ & 0.4 & -0.003 & $-0.008,0.002$ & 0.2 \\
\hline Casein (g/d) & -0.0004 & $-0.002,0.001$ & 0.5 & -0.0006 & $-0.002,0.001$ & 0.5 \\
\hline $\mathrm{Ca}$ intake within recommendations & -0.085 & $-0.135,-0.035$ & 0.001 & -0.088 & $-0.139,-0.037$ & 0.001 \\
\hline Vitamin $D \geq 5.8 \mu \mathrm{g} / \mathrm{d}$ & 0.004 & $-0.041,0.050$ & 0.8 & -0.001 & $-0.047,0.045$ & 0.9 \\
\hline Whey $\geq 9.6 \mathrm{~g} / \mathrm{d}$ & -0.050 & $-0.096,-0.005$ & 0.031 & -0.056 & $-0.102,-0.010$ & 0.017 \\
\hline Casein $\geq 32.0 \mathrm{~g} / \mathrm{d}$ & -0.042 & $-0.088,0.003$ & 0.07 & -0.048 & $-0.095,-0.002$ & 0.04 \\
\hline
\end{tabular}

*Linear regression models adjusted for age, gender, education, smoking, sucrose intake, alcohol consumption, number of teeth, daily brushing, regular visits to the dentist and chronic illness. Analysis performed with 3287 observations.

report alcohol consumption above the recommendation, whey intake below the median and higher sucrose consumption, compared with those without periodontitis.

Table 2 shows the associations between intakes of $\mathrm{Ca}$, vitamin $\mathrm{D}$, casein and whey (both as continuous and binary variables) and severe periodontitis. In the crude models none of the nutrients was significantly associated with periodontitis, except whey protein intake $\geq 9.6 \mathrm{~g} / \mathrm{d}$. However, when the analyses were adjusted for age, gender, education, smoking, sucrose intake, alcohol consumption, number of teeth, daily brushing, regular visits to the dentist and chronic illness, modest inverse associations between intakes of $\mathrm{Ca}$ within recommendations $(\mathrm{OR}=$ 0.76; $95 \%$ CI 0.58, 0.99), whey $\geq 9.6 \mathrm{~g} / \mathrm{d}(\mathrm{OR}=0.75 ; 95 \%$ CI $0.58,0.97)$ and casein $\geq 32 \mathrm{~g} / \mathrm{d}(\mathrm{OR}=0.75 ; 95 \% \mathrm{CI} 0.58$, 0.97) and severe periodontitis were observed. $\mathrm{Ca}(\mathrm{mg} / \mathrm{d})$, whey $(\mathrm{g} / \mathrm{d})$ and casein $(\mathrm{g} / \mathrm{d})$ intakes as continuous variables remained non-significant after adjustments and vitamin $\mathrm{D}$ intake was not directly associated with severe periodontitis.

Since vitamin $\mathrm{D}$ is essential for Ca absorption ${ }^{(13)}$, analyses regarding $\mathrm{Ca}$ intake were stratified by vitamin $\mathrm{D}$ intakes (within $v$, above the 50th percentile), but no interaction was found (data not shown). Furthermore, interactions between whey, casein and vitamin D intakes were explored via stratified analyses, but no significant results were observed. Associations did not differ according to vitamin D intake levels.

Associations between intakes of $\mathrm{Ca}$, vitamin $\mathrm{D}$, casein and whey and mean CAL showed the same pattern of results as for severe periodontitis (Table 3). No difference in the associations was found when the mean CAL included or excluded mid-facial and mid-lingual sites, respectively.

\section{Discussion}

Our findings showed that higher intakes of $\mathrm{Ca}$ (i.e. within current recommendations) and of whey protein and casein were individually associated with a lower occurrence of severe periodontitis, after adjustments for age, gender, education, smoking, sucrose intake, alcohol consumption, number of teeth, daily brushing, regular visits to the dentist and chronic illness, in a subgroup of participants in whom dental health was examined, but vitamin D intake was not directly associated with periodontitis.

To our knowledge, the present study is the first observational one examining associations between intakes of casein and whey protein and periodontitis, and therefore it cannot be compared with other data. However, previous 
studies have shown significant inverse associations between dietary $\mathrm{Ca}$ intake and periodontitis ${ }^{(7,14)}$.

Activated vitamin D (i.e. 1,25-dihydroxyvitamin D $\left.\left(1,25(\mathrm{OH})_{2} \mathrm{D}\right)\right)$ plays a role in maintaining oral health through its effects on $\mathrm{Ca}$ and bone metabolism, bacterial suppression and innate immunity ${ }^{(8,29)}$. Although cholecalciferol (vitamin $\mathrm{D}_{3}$ ) is produced endogenously upon UV-B sunlight irradiation of the skin, some individuals have a greater need for dietary and supplemental sources $^{(13)}$, such as elderly and Nordic populations, who are less exposed to sunlight or less efficient in generating endogenous vitamin $\mathrm{D}^{(30)}$. The present study showed that vitamin $\mathrm{D}$ intake alone was not associated with periodontitis. Vitamin D status influences intestinal Ca absorption $^{(13)}$. However, level of vitamin D intake was not found to be an important effect modifier in the associations between $\mathrm{Ca}$ and whey intakes and periodontitis risk in the present cohort, although in our previous study it was observed that intake of $\mathrm{Ca}$ and dairy servings within recommendations were inversely associated with oral plaque score among those with higher, but not lower, vitamin D intake ${ }^{(18)}$; possibly because vitamin D intakes were lower in the present study than in our earlier study $^{(18)}$. This issue requires further investigation in cohorts with a wider range of vitamin D intakes. While we did not measure serum concentrations of 25-hydroxyvitamin D (25(OH)D; or vitamin D status), it would be expected that vitamin D intake would be correlated with vitamin D status, especially in Nordic countries, where exposure to sunlight is limited to summer. Vitamin D deficiency is a risk factor for several disorders with inflammatory components and one randomized clinical trial showed reductions in inflammatory markers (matrix metalloproteinases MMP9 and MMP2; C-reactive protein) with higher vitamin D status at baseline and after supplementation ${ }^{(31)}$.

Low vitamin D status is prevalent in Western countries; according to the Danish Health and Medical Authority, approximately half of the Danish adult population have vitamin $\mathrm{D}$ deficiency (serum $25(\mathrm{OH}) \mathrm{D}<25 \mathrm{nmol} / \mathrm{l}$ ). In the present study population, only $33.2 \%$ and $7 \cdot 8 \%$ of participants reported $\mathrm{Ca}$ and vitamin $\mathrm{D}$ intakes that were within current recommendations, respectively. In 2011, Denmark started voluntary fortification of milk with vitamin $\mathrm{D}$, but this did not affect the estimations of dietary vitamin D intake in the current study as it was carried out prior to implementation of this fortification policy.

Although there is no universally established case definition for chronic periodontitis in clinical and epidemiological studies, the definition proposed by the CDC/AAP ${ }^{(26)}$ has become more widely used and its utilization in the present study will allow comparisons with other studies in the future. Mean CAL was calculated including and excluding mid-facial and mid-lingual sites. The rationale for this was based on the fact that mid-facial recession commonly occurs in isolation and not necessarily in relation to periodontitis ${ }^{(32)}$.
Our findings consistently showed inverse associations between intakes of $\mathrm{Ca}$ within the recommendations, casein $\geq 32.0 \mathrm{~g} / \mathrm{d}$ and whey protein $\geq 9.6 \mathrm{~g} / \mathrm{d}$ and both measures of mean CAL (including and excluding midfacial and mid-lingual sites). However, these associations were notably weak and therefore of limited clinical relevance. When the main exposure variables $(\mathrm{Ca}$, vitamin $\mathrm{D}$, casein and whey) were used as continuous variables in the models, no associations were found.

For severe periodontitis, in the fully adjusted models, associations were modest when exposure variables were categorized by specific levels of intake. However, intakes of $\mathrm{Ca}(\mathrm{mg} / \mathrm{d})$, casein $(\mathrm{g} / \mathrm{d})$ and whey $(\mathrm{g} / \mathrm{d})$ as continuous variables were weakly associated with periodontitis. Periodontitis is a multifactorial condition; thus a single risk factor (especially dietary factors) would play a modest role in the disease progression. One potential reason for such findings could be that our case definition was based on severe periodontitis and severe cases might be the result of genetic factors and severe inflammatory diseases ${ }^{(33)}$, rather than low intakes of $\mathrm{Ca}$, casein and whey alone. Apart from that, given the apparent chronic nature of periodontitis and of alveolar bone resorption, several years of follow-up may be necessary to detect beneficial longterm effects of $\mathrm{Ca}$, casein and whey intakes on periodontitis. Therefore, one of the major limitations of the present study is its cross-sectional design.

In the present study, periodontal examinations were performed in one randomly selected upper and lower quadrant. In total, $9.6 \%$ of the present study population had periodontitis according to the CDC/AAP definition ${ }^{(26)}$. Although full mouth examination is preferable, time limitations for the clinical examinations resulted in the decision to use partial recording. It has been estimated that a partial recording protocol similar to that used in the present study, with random sampling of one maxillary and one mandibular quadrant evaluating CAL at six sites per tooth, reveals $5 \%$ fewer individuals with periodontitis compared with full mouth registrations ${ }^{(34)}$. This might indicate that associations presented in the study could have been somewhat underestimated.

Another limitation of the current study was the lack of detailed information on $\mathrm{Ca}$ and vitamin $\mathrm{D}$ supplementation, resulting in possible underestimation of $\mathrm{Ca}$ and vitamin $\mathrm{D}$ intakes and consequently underestimation of our findings for these variables. In addition, the study was hampered by exclusion of participants due to insufficient information on dietary intake, clinical periodontal examination or other covariates (in the 3287 participants with complete data sets, out of 18065 participants). However, when responders and non-responders were compared, no significant differences in sex ratio or age were observed. Responders were more likely to report higher income and more years of education so that the presence of selection bias, or the 'healthy participant effect', cannot be entirely ruled out; and if responders tended to belong to a low-risk 
group this would have led to underestimation of the strength of our findings. Furthermore, it was not possible to explore the role of ethnicity in these associations as approximately $95 \%$ of the sample was born in Denmark and $92 \%$ of their parents were Danish.

Habitual dietary intake can only be assessed by asking individuals to report their intake. Hence, all dietary methods, including the FFQ, rely on memory, willingness to report actual intake and ability to generalize intake, and are therefore subject to recall bias ${ }^{(35)}$. Such biased reporting seems particularly liable to cause underestimation of consumption of food items rich in fat and/or simple carbohydrates and overestimation of intakes of healthy foods, such as fruits and vegetables, and potentially of some dairy products ${ }^{(35,36)}$. In the present study, therefore, we cannot exclude the possibility that reporting bias contributed to the finding that $\mathrm{Ca}$ intake within recommendations was inversely associated with severe periodontitis.

The use of a large population including both men and women in a wide age range (18-95 years), with detailed information in respect of both medical and dental outcomes and risk factors, is one of the strengths of the present study. Therefore, our results may be generalizable to other Western populations.

In conclusion, our cross-sectional study findings corroborate the hypothesis that higher $\mathrm{Ca}$, casein and whey intakes are associated with lower periodontitis risk, although it is not possible to infer causality from these cross-sectional associations. However, adherence to the suggested recommendations for Ca intake may not only have a beneficial impact on bone health but also on oral health and other chronic diseases. Therefore, consumption of foods rich in Ca (e.g. dairy foods) should be promoted, as this could contribute to prevention of periodontitis. Given that periodontitis is a chronic condition that develops over time, further longitudinal studies should be conducted to explore these findings more rigorously.

\section{Acknowledgements}

Acknowledgements: The authors thank the study participants for providing the data and Claus Host and Thorhallur Ingi Halldorsson for their assistance with the dietary database. Financial support: The present study was supported by the Danish Dental Association; the Danish Foundation for Mutual Efforts in Dental Care (grant number RLTN5530 - 08/2405); TrygFonden (grant number 1140-09); and the Health Insurance Foundation (grant number 2007B132). The funders had no role in the design, analysis or writing of this article. Conflict of interest: None. Authorship: A.R.A.A. was the lead author of the paper; N.-E.F. was the principal investigator of the oral health part of the DANHES 2007-2008 and the chair of data collection. B.J.B., L.B.C., J.K., N.-E.F. and B.L.H. provided content and editorial contribution; J.K. and B.L.H. and L.B.C. provided data quality assistance. Ethics of human subject participation: The study was conducted in accordance with the Helsinki Declaration and approved by the Ethical Committees for the Region of Copenhagen. Written informed consent was obtained from all participants.

\section{References}

1. Graves DT, Oates T \& Garlet GP (2011) Review of osteoimmunology and the host response in endodontic and periodontal lesions. J Oral Microbiol 3, doi: 10.3402/jom. v3i0.5304

2. Pihlstrom BL, Michalowicz BS \& Johnson NW (2005) Periodontal diseases. Lancet 366, 1809-1820.

3. Al-Zahrani MS, Borawski EA \& Bissada NF (2005) Increased physical activity reduces prevalence of periodontitis. $J$ Dent 33, 703-710.

4. Kongstad J, Hvidtfeldt UA, Gronbaek M et al. (2008) Amount and type of alcohol and periodontitis in the Copenhagen City Heart Study. J Clin Periodontol 35, 1032-1039.

5. Kongstad J, Hvidtfeldt UA, Gronbaek M et al. (2009) The relationship between body mass index and periodontitis in the Copenhagen City Heart Study. J Periodontol 80, 1246-1253.

6. Alshouibi EN, Kaye EK, Cabral HJ et al. (2013) Vitamin D and periodontal health in older men. J Dent Res 92, 689-693.

7. Al-Zahrani MS (2006) Increased intake of dairy products is related to lower periodontitis prevalence. J Periodontol 77, 289-294.

8. Boyd LD \& Madden TE (2003) Nutrition, infection, and periodontal disease. Dent Clin North Am 47, 337-354.

9. Bowden GH \& Li YH (1997) Nutritional influences on biofilm development. Adv Dent Res 11, 81-99.

10. Boyd LD \& Lampi KJ (2001) Importance of nutrition for optimum health of the periodontium. J Contemp Dent Pract 2, 36-45.

11. Al-Zahrani MS, Borawski EA \& Bissada NF (2005) Periodontitis and three health-enhancing behaviors: maintaining normal weight, engaging in recommended level of exercise, and consuming a high-quality diet. J Periodontol 76, 1362-1366.

12. Moynihan PJ (2005) The role of diet and nutrition in the etiology and prevention of oral diseases. Bull World Health Organ 83, 694-699.

13. Christakos S, Dhawan P, Porta A et al. (2011) Vitamin D and intestinal calcium absorption. Mol Cell Endocrinol 347, 25-29.

14. Adegboye AR, Christensen LB, Holm-Pedersen $\mathrm{P}$ et al. (2012) Intake of dairy products in relation to periodontitis in older Danish adults. Nutrients 4, 1219-1229.

15. Danielsson Niemi L, Hernell O \& Johansson I (2009) Human milk compounds inhibiting adhesion of mutans streptococci to host ligand-coated hydroxyapatite in vitro. Caries Res $\mathbf{4 3}$, 171-178.

16. Krall EA, Wehler C, Garcia RI et al. (2001) Calcium and vitamin D supplements reduce tooth loss in the elderly. Am $J$ Med 111, 452-456.

17. Krissansen GW (2007) Emerging health properties of whey proteins and their clinical implications. J Am Coll Nutr 26, issue 6, 713S-723S.

18. Adegboye AR, Christensen LB, Holm-Pedersen $\mathrm{P}$ et al. (2013) Intakes of calcium, vitamin D, and dairy servings and dental plaque in older Danish adults. Nutr J 12, 61.

19. Eriksen L, Gronbaek M, Helge JW et al. (2011) The Danish Health Examination Survey 2007-2008 (DANHES 2007-2008). Scand J Public Health 39, 203-211.

20. Kongstad J, Ekstrand K, Qvist V et al. (2013) Findings from the oral health study of the Danish Health Examination Survey 2007-2008. Acta Odontol Scand 71, 1560-1569. 
21. Tjonneland A, Overvad K, Haraldsdottir J et al. (1991) Validation of a semiquantitative food frequency questionnaire developed in Denmark. Int J Epidemiol 20, 906-912.

22. Tjonneland A, Haraldsdottir J, Overvad K et al. (1992) Influence of individually estimated portion size data on the validity of a semiquantitative food frequency questionnaire. Int J Epidemiol 21, 770-777.

23. Lauritsen J (1999) FoodCalc ${ }^{\circledR}$. http://www.ibt.ku.dk/jesper/ foodcalc/ (accessed August 2014).

24. Ross CA, Taylor CL, Yaktine AL et al. (2011) Dietary Reference Intakes for Calcium and Vitamin D. Washington, DC: The National Academies Press.

25. The Dairy Council (2011) Nutrients and dairy. http://www. milk.co.uk/ (accessed August 2014).

26. Page RC \& Eke PI (2007) Case definitions for use in population-based surveillance of periodontitis. J Periodontol 78, 1387-1399.

27. National Board of Health (2014) Alcohol. http://sundheds styrelsen.dk/en/health/alcohol (accessed August 2014).

28. Yudkin J (1990) Report of the COMA panel on dietary sugars and human disease: discussion paper. JR Soc Med 83, 627-628.

29. Grant WB \& Boucher BJ (2010) Are Hill's criteria for causality satisfied for vitamin D and periodontal disease? Dermatoendocrinology 2, 30-36.
30. Hilger J, Friedel A, Herr R et al. (2014) A systematic review of vitamin D status in populations worldwide. BrJ Nutr 111, $23-45$.

31. Timms PM, Mannan N, Hitman GA et al. (2002) Circulating MMP9, vitamin D and variation in the TIMP-1 response with VDR genotype: mechanisms for inflammatory damage in chronic disorders? OJM 95, 787-796.

32. Heasman PA, Holliday R, Bryant A et al. (2015) Evidence for the occurrence of gingival recession and non-carious cervical lesions as a consequence of traumatic tooth brushing. J Clin Periodontol 42, Suppl. 16, S237-S255.

33. Stabholz A, Soskolne WA \& Shapira L (2010) Genetic and environmental risk factors for chronic periodontitis and aggressive periodontitis. Periodontol $2000 \mathbf{5 3}$, 138-153.

34. Susin C, Kingman A \& Albandar JM (2005) Effect of partial recording protocols on estimates of prevalence of periodontal disease. J Periodontol 76, 262-267.

35. Lissner L, Heitmann BL \& Lindroos AK (1998) Measuring intake in free-living human subjects: a question of bias. Proc Nutr Soc 57, 333-339.

36. Heitmann BL, Lissner L \& Osler M (2000) Do we eat less fat, or just report so? Int J Obes Relat Metab Disord 24, 435-442. 